\title{
Bisfosfonatos e a osseointegração
}

\author{
Bisphosphonates and osseointegration
}

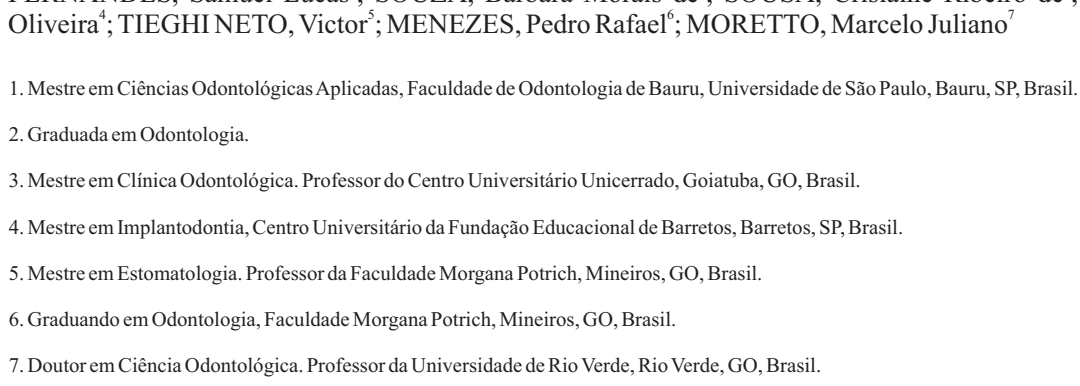

Recebido: 08.05.2017

Aceito: 30.06 .2017

\section{RESUMO}

Os bisfosfonatos são um grupo de drogas comumente prescritos para tratar pacientes portadores de doenças ósseas metabólicas e oncológicas. E casos de osteonecrose em mandíbula são descritos, relatando essa patologia em indivíduos usuários de bisfosfonatos após procedimentos de cirurgia oral, incluindo a colocação de implantes dentários. Esta revisão tem como objetivo dar um norte ao clínico sobre a atual perspectiva da relação entre bisfosfonatos e implantes dentários. Para tal, toma por base estudos e relatos de casos clínicos realizados em seres humanos, a fim de avaliar se pacientes em terapia com bisfosfonatos ou que já se submeteram a ela são candidatos a receberem implantes dentários, bem como se forem submetidos, qual o grau de risco de desenvolver osteonecrose de mandíbula. A literatura atual mostra um índice de sucesso considerável dentre os pacientes usuários de bisfosfonatos e que receberam implantes dentários, entretanto é importante que o cirurgião dentista tome algumas precauções e alerte o paciente sobre os riscos da perda do implante e da necrose dos maxilares em função do procedimento executado.

Palavras-chave: Implantes dentários. Difosfonatos. Osteonecrose.

\begin{abstract}
Bisphosphonates are a group of drugs commonly prescribed to treat patients with metabolic and oncological bone diseases. Moreover, cases of osteonecrosis in the mandible described, reporting this pathology in individuals using bisphosphonates following oral surgery procedures, including placement of dental implants. This review aims to give the clinician an insight into the current perspective of the relationship between bisphosphonates and dental implants. To this end, it is based on studies and reports of clinical cases carried out in humans in order to assess whether patients on bisphosphonates therapy or who have already undergone it are candidates to receive dental implants, and if they are submitted, what degree of risk to develop mandibular osteonecrosis. The current literature shows a considerable success rate among patients with bisphosphonates and who received dental implants; however, it is important that the dental surgeon take some precautions and alert the patient about the risks of implant loss, maxillary necrosis due to performed.
\end{abstract}

Keywords: Dental implants. Diphosphonates. Osteonecrosis. 


\section{INTRODUÇÃO}

A osseointegração é definida como uma conexão direta entre a superfície do implante e o osso, em que a peça foi implantada, no caso da odontologia, o pino do implante, que será submetido a uma carga oclusal. Dentre os principais aspectos de risco que podem interferir no estabelecimento da osseointegração, destacam-se o tabagismo, a radioterapia, o diabetes, a doença periodontal ativa, a osteoporose, a idade e a densidade óssea deficiente ${ }^{2-}$ ${ }^{4}$. Alguns medicamentos também requerem atenção antes da colocação de implantes, como os bisfosfonatos.

Os bisfosfonatos são fármacos utilizados no tratamento de várias doenças ósseas, como a osteoporose. Apresentamse sob duas formas: com e sem nitrogênio em sua composiçãos. Eles atuam nos processos de reabsorção e remodelação óssea, por via de uma redução da atividade dos osteoclastos, levando a uma diminuição da taxa de reabsorção do osso. Apesar da principal função dos bisfosfonatos ser a inibição da reabsorção óssea, como efeito colateral leva também a uma diminuição da renovação e remodelação óssea .

Nos últimos anos, houve um aumento do número da utilização de implantes dentários, sendo que um grande percentual dos pacientes submetidos à cirurgia para a inserção de implantes está em uma faixa etária superior aos sessenta anos de idade $e^{3,7-8}$. Idade essa em que estão mais vulneráveis à osteoporose e a outras doenças osteodegenerativas?

Embora haja muitos relatos sobre os fatores de risco para o desenvolvimento da osteonecrose associada ao bisfosfonato, o papel do implante dentário como um fator de risco local ainda é muito discutido". Independente da alteração encontrada, o cirurgião dentista implantodontista deve estar apto a reconhecer as alterações e tratá-las de forma singular ${ }^{6}$.

Visto que é um tema controverso na literatura, o objetivo desse trabalho foi realizar uma revisão da literatura por meio de estudos clínicos, a fim de nortear o clínico quanto à previsibilidade e à segurança da realização de implantes dentários em pacientes que fazem uso de bisfosfonatos.

\section{REVISÃO DE LITERATURA}

A perda de implantes dentários relacionada com a osteoporose também foi investigada por vários autores, em que esses estudos mostraram taxas altas de sucesso do implante, sem aparente contraindicação, nesses pacientes. Porém, a relação entre o uso de bisfosfonatos e o sucesso dos implantes ainda não foram investigados de forma minuciosa $^{8,10}$.
Tabela 1 - Estudos que relacionam o uso de bisfosfonatos e o índice de sucesso do implante ósseo integrado.

\begin{tabular}{|c|c|c|c|c|c|c|c|}
\hline Autor/ano & $\begin{array}{c}\text { Número } \\
\text { de } \\
\text { pacientes }\end{array}$ & Sexo & Idade & Medicação & $\begin{array}{c}\text { Número de } \\
\text { Implantes/região }\end{array}$ & $\begin{array}{l}\text { Tempo de } \\
\text { aguardo }\end{array}$ & Sucesso \\
\hline $\begin{array}{l}\text { MANFREDI et } \\
\text { al. }(2011)^{3}\end{array}$ & 25 & $\mathrm{~F}$ & $\begin{array}{l}70.4 \\
\text { anos }\end{array}$ & $\begin{array}{l}\text { Alendronato } \\
\text { Clorodrato } \\
\text { Ibandronato } \\
\text { Neridronato }\end{array}$ & $\begin{array}{l}\begin{array}{l}\text { Mandibula e } \\
\text { maxila }\end{array} \\
\end{array}$ & 3 meses & $72,72 \%$ \\
\hline $\begin{array}{l}\text { ZAHID et al. } \\
(2011)^{8}\end{array}$ & 300 & $\begin{array}{l}\mathrm{F} \\
\mathrm{M}\end{array}$ & 56 anos & $\begin{array}{l}\text { Ibandronato } \\
\text { de sódio } \\
\text { Alendronato }\end{array}$ & $\begin{array}{l}66 \\
\text { Mandibula }\end{array}$ & zero & $97.10 \%$ \\
\hline $\begin{array}{l}\text { YIP et al. } \\
(2012)^{4}\end{array}$ & 337 & $\mathrm{~F}$ & 40 anos & $\begin{array}{l}\text { Alendronato } \\
\text { Risedronato; } \\
\text { Ibandronato } \\
\text { Etidronato }\end{array}$ & $\begin{array}{l}118 \\
\text { Mandibula }\end{array}$ & $\begin{array}{l}\text { Durante o uso } \\
\text { da medicação }\end{array}$ & $66.17 \%$ \\
\hline $\begin{array}{l}\text { KWON et al. } \\
(2012)^{11}\end{array}$ & $\begin{array}{l}17 \mathrm{~F} \\
2 \mathrm{M}\end{array}$ & $\begin{array}{l}\mathrm{F} \\
\mathrm{M}\end{array}$ & $\begin{array}{l}42-81 \\
\text { anos }\end{array}$ & $\begin{array}{l}\text { Alendronato } \\
\text { Zolendronato } \\
\text { Ibandronato } \\
\text { Risedronato } \\
\text { Pamidronato }\end{array}$ & $\begin{array}{l}\text { Mandibula e } \\
\text { maxila }\end{array}$ & Zero & $47.37 \%$ \\
\hline $\begin{array}{l}\text { OLIVEIRA et } \\
\text { al. }(2014)^{12}\end{array}$ & 45 & $\mathrm{~F}$ & $\begin{array}{l}61.6 \pm \\
5.97 \\
\text { anos }\end{array}$ & $\begin{array}{l}\text { Alendronato } \\
\text { de sódio }\end{array}$ & Mandibula & $\begin{array}{l}\text { Colocou o } \\
\text { implante } \\
\text { durante o uso } \\
\text { da medicaçăo }\end{array}$ & $100 \%$ \\
\hline $\begin{array}{l}\text { SIEBERT et } \\
\text { al. (2015) }\end{array}$ & 24 & $F$ & $\begin{array}{c}54 \\
\text { anos }\end{array}$ & $\begin{array}{l}\text { Ácido } \\
\text { zoledrônico }\end{array}$ & $\begin{array}{l}120 \\
\text { Mandibula }\end{array}$ & $\begin{array}{l}\text { Usou } \\
\text { bisfosfonatos } \\
\text { por } 2 \text { a } 3 \text { anos } \\
\text { antes da } \\
\text { implantação }\end{array}$ & $100 \%$ \\
\hline $\begin{array}{l}\text { FAMINI et al. } \\
(2015)^{14}\end{array}$ & 30 & $\begin{array}{l}F \\
M\end{array}$ & $\begin{array}{c}50-80 \\
\text { anos }\end{array}$ & $\begin{array}{l}\text { Fosamax } \\
\text { Fortical } \\
\text { Forteo }\end{array}$ & $\begin{array}{l}31 \\
\text { Maxila Mandibula }\end{array}$ & $\begin{array}{l}\text { Durante o uso } \\
\text { da medicação }\end{array}$ & $100 \%$ \\
\hline $\begin{array}{l}\text { JEFFCOAT } \\
(2006)^{15^{15}}\end{array}$ & 50 & $\begin{array}{l}\mathrm{F} \\
\mathrm{M}\end{array}$ & $\begin{array}{c}\begin{array}{c}30 \text { a } 79 \\
\text { anos }\end{array} \\
\text {. }\end{array}$ & $\begin{array}{l}\text { Alendronato } \\
\text { Risendronato }\end{array}$ & 210 & $\begin{array}{l}\text { Durante o uso } \\
\text { da medicação }\end{array}$ & $\begin{array}{l}\text { Maior que } \\
99 \%\end{array}$ \\
\hline $\begin{array}{l}\text { MOZZATTI et } \\
\text { al. }(2015)^{16}\end{array}$ & 235 & $F$ & & & & & $98.7 \%$ \\
\hline
\end{tabular}

\section{DISCUSSÃ0}

A osseointegração é uma condição imprescindível para o sucesso dos implantes dentários. Essa que é definida por Brånemark ${ }^{1}$ como uma conexão direta entre a superfície do implante e o osso do indivíduo que o recebeu. O metal que se mostra com melhores propriedades físicas e biológicas é o titânio ${ }^{17}$. E a cada dia vem se buscando mais alternativas para otimizar o processo de osseointegração.

Com os implantes se popularizando a cada dia, os insucessos também têm acompanhado nas devidas proporções, e entre os diversos fatores que podem ser associados à osseointegração se apresentam como um dos principais contribuintes para as taxas de insucesso ${ }^{18}$. São inúmeros os fatores etiológicos que contribuem para falhas na osseointegração, podendo advir de causas sistêmicas e/ou locaiis ${ }^{18-20}$, entretanto é necessário que se interprete as informações com cautela, uma vez que nem sempre a etiologia é bem descrita nos estudos, apresentando muitas vezes dados confusos e desconexos em relação às falhas ${ }^{21}$.

Dentre as causas sistêmicas mais atribuídas ao insucesso dos implantes dentários se destaca a osteoporose $^{20,22}$, doenças cardiovasculares ${ }^{20}$, diabetes ${ }^{20,23}$, hipotireoidismo ${ }^{20}$, Doença de $\mathrm{Crohn}^{22-23}$, problemas gastrointestinais ${ }^{23}$, pacientes que fazem reposição hormonal $^{23}$ e o hábito de fumar ${ }^{20,22-23}$, sendo o tabagismo um dos fatores que mais influenciam negativamente no índice de sucesso ${ }^{20,22-24}$.

Já dentre os fatores locais, a condição da saúde bucal do indivíduo, a presença de dentes adjacentes22-23, presença 
de lesões periapicais23, largura e comprimento dos implantes22 e locais de inserção do implante22-23 irão contribuir para o sucesso ou a falha do tratamento.

Há vários relatos que demonstraram sucesso na colocação do implante em pacientes que tomam bisfosfonatos8,12-16,19. Porém, a relação entre o uso de bisfosfonatos e o sucesso dos implantes ainda não foram investigados de forma minuciosa e criteriosa8,10. Uma vez que se pode observar discrepâncias de índices de sucesso e de osteonecrose dos maxilares relacionadas à colocação de implantes, os resultados muitas vezes não levam em conta a abordagem multifatorial do insucesso.

Sabe-se que a colocação de implantes dentários induz a uma série de alterações metabólicas ao redor dos implantes devendo levar à formação de osso intrinsecamente ligado à superfície do mesmo. Se o osso que circunda o implante apresenta alta concentração de bisfosfonatos, a remodelação óssea será prejudicada, tendo assim, risco de necrose ao redor do osso19. A osteonecrose dos maxilares irá decorrer da inatividade osteoclástica, promovida pelos bisfosfonatos que irão atuar na fisiologia celular dos osteoclastos, afetando assim a remodelação óssea. Essa inatividade irá levar a não reabsorção do osso necrosado, afetando ainda mais a nutrição e promovendo a necrose dos maxilares 19,25.

Vários relatos de osteonecrose dos maxilares associada ao uso de bisfosfonatos, apresenta predileção na mandíbula. A ação do bisfosfonato é fortalecer a matriz óssea, tornando-a mais resistente às fraturas. Porém, a formação excessiva de osso cortical faz com que a mandíbula fique com pouca nutrição via osso medular. Então a única fonte de nutrição do osso cortical é fornecida pelo periósteo. Com pouca ou sem fonte de nutrição após um procedimento cirúrgico, o osso tende a necrosar 26.

Diversos tipos de bisfosfonatos estão disponíveis no mercado, como o Alendronato, Zolendronato, Ibandronato, Risedronato, Pamidronato, Etidronato, Clorodrato e Neridronato, todavia pode-se verificar que alguns são mais prescritos que outros. Segundo alguns estudos, pode-se observar que o Alendronato3,11,12 é o mais utilizado. Essas medicações podem ser administradas via oral ou intravenosa, sendo que a utilização intravenosa de bisfosfonatos tipo IV, está ligada a bisfosfonatos mais potentes e consideradas com maior potencial de indução da osteonecrose por bisfosfonatos 19,27. Por meio de diversos estudos, o que se pode observar é que os pacientes que mais fazem uso dessa medicação são mulheres em uma faixa etária acima dos 50 anos de idade3-4,8,11-16.

A realização de implantes em pacientes usuários de bisfosfonatos ainda é uma área obscura e depende de muitos fatores. Entretanto, alguns autores28-29 afirmam que um histórico de uso oral ou intravenoso de bisfosfonatos não é uma contraindicação absoluta para a colocação de implantes dentários, uma vez que estudos8,12-16 mostram a colocação de implantes com sucesso nesta população de pacientes. Já alguns mostram um índice de falha preocupante $3-4,11$, com até $50 \%$ de insucessos na colocação de implantes em pacientes que fazem uso dos bisfosfonatos 11. Apesar disso tem-se recomendações para a realização de implantes nesses pacientes 28 .

Deve-se, no entanto, ponderar esses estudos uma vez que a falta de informações adicionais nos deixa à mercê da interpretação de dados, com fraca evidência científica e que pode levar-nos a resultados pouco conclusivos. Ora, pois então cabe ao cirurgião dentista observar o paciente de forma integral, verificando sua condição sistêmica, capacidade de reparo, genética, anatomia dos maxilares, tempo de uso da medicação e de acordo com os dados obtidos, proceder ou não a colocação de implantes neste paciente, uma vez que estas variáveis estão diretamente ligadas ao potencial de osteonecrose dos maxilares induzida por bisfosfonatos 30 .

Recomenda-se, se o paciente estiver tomando medicamento por mais de 3 anos, interromper o uso por pelo menos 3 meses antes da realização de qualquer procedimento cirúrgico. Lembrando que a descontinuação do tratamento com bisfosfonatos não deve ser feita isoladamente pelo cirurgião dentista, e sim, pelo médico que prescreveu o medicamento, sendo que o paciente deve estar ciente de qualquer risco e benefício ligados à interrupção do bisfosfonato 7,31. Recomenda-se ainda que o paciente aguarde o tempo de recuperação cirúrgica para que volte a fazer o uso da medicação.

\section{CONCLUSÃO}

Mesmo que os índices de sucesso de implantes osseointegrados em pacientes que fazem uso de bisfosfonatos sejam semelhantes aos de pacientes saudáveis, espera-se que os cirurgiões dentistas estejam cientes de que pacientes que fazem o uso desses medicamentos terão interferências na remodelação óssea. Demanda-se, portanto, que se esclareça ao paciente sobre os riscos, e que se tenha o menor número possível de outros fatores sistêmicos associados para uma melhor previsibilidade, além de se ter mais cuidados prévios, durante e no pós-operatório, buscando informar os riscos do procedimento ao paciente.

\section{REFERÊNCIAS}

1. Brånemark PI, Adell R, Breine U, Hansson BO, Lindström J, Ohlsson A. Intra-osseous anchorage of dental prostheses. I. Experimental studies. Scand J Plast Reconstr Surg. 1969;3(2):81-100. 
2. Zavanelli RA, Guilherme AS, Castro AT, Fernandes JMA, Pereira RE, Garcia RR. Fatores locais e sistêmicos relacionados aos pacientes que podem afetar a osseointegração. RGO, 2011;59:133-46.

3. Manfredi M, Merigo E, Guidotti R, Meleti M, Vescovi P. Bisphosphonate-related osteonecrosis of the jaws: a case series of 25 patients affected by osteoporosis. Int J Oral Maxillofac Surg. 2011;40(3):277-84.

4. Yip JK, Borrell LN, Cho SC, Francisco H, Tarnow DP. Association between oral bisphosphonate use and dental implant failure among middle-aged women. J Clin Periodontol. 2012;39(4):408-14.

5. Izquierdo CM, Oliveira MGE, Weber JBB. Terapêutica com bisfosfonatos: implicações no paciente odontológico - revisão de literatura. Rev Faculdade Odontol Passo Fundo. 2011;16(3):347-52.

6. Abdou WM, Shaddy AA. The development of bisphosphonates for therapeutic uses, and bisphosphonate structure activity consideration. Eurasian Conference Heterocycl Chem. 2009;5:143-82.

7. Serra MP, Llorca CS, Donat FJ. Oral implants in patients receiving bisphosphonates: a review and update. Med Oral Patol Oral Cir Bucal. 2008;13(12):E755-60.

8. Zahid TM, Wang BY, Cohen RE. Influence of bisphosphonates on alveolar bone loss aroundosseointegrated implants. J Oral Implantol. 2011;37(3):335-46.

9. Jacobsen C, Metzler P, Rössle M, Obwegeser J, Zemann W, Grätz KW. Osteopathology induced by bisphosphonates and dental implants: clinical observations. Clin Oral Investig. 2013;17(1):167-75.

10. Faverani LP, Ramalho-Ferreira G, Gaetti-Jardim EC, Okamoto R, Shinohara EH, Assunção WG, et al. Implantes osseointegrados: evolução sucesso. Salusvita. 2011;30(1):47-58.

11. Kwon TG, Lee CO, Park JW, Choi SY, Rijal G, Shin HI. Osteonecrosis associated with dental implants in patients undergoing bisphosphonate treatment. Clin Oral Implants Res. 2014;25(5):632-40.

12. Oliveira PS, Rodrigues JA, Shibli JA, Piattelli A, Iezzi G, Perrotti V. Influence of osteoporosis on the osteocyte density of human mandibular bone samples: a controlled histological human study. Clin Oral Implants Res. 2016;27(3):325-8.

13. Siebert T, Jurkovic R, Statelova D, Strecha J. Immediate implant placement in a patient with osteoporosis undergoing bisphosphonate therapy: 1-year preliminary prospective study. J Oral Implantol. 2015;41(Spec N):360-5

14. Famili P, Zavoral JM. Low skeletal bone mineral density does not affect dental implants. J Oral Implantol. 2015;41(5):550-3.

15. Jeffcoat MK. Safety of oral bisphosphonates: controlled studies on alveolar bone. Int J Oral Maxillofac Implants. 2006;21(3):349-53.

16. Mozzati M, Arata V, Giacomello M, Del Fabbro M, Gallesio G, Mortellaro C, et al. Failure risk estimates after dental implants placement associated with plasma rich in growth factor-Endoret in osteoporotic women under bisphosphonate therapy. J Craniofac Surg. 2015;26(3):749-55.

17. Brånemark PI, Hansson BO, Adell R, Breine U, Lindström J, Hallén $\mathrm{O}$, et al. A. Osseointegrated implants in the treatment of the edentulous jaw. Experience from a 10-year period. Scand J Plast Reconstr Surg. 1977;16 Suppl:1-132.

18. Esposito M, Hirsch JM, Lekholm U, Thomsen P. Biological factors contributing to failures of osseointegrated oral implants. (II).
Etiopathogenesis. Eur J Oral Sci. 1998;106(3):721-64.

19. Kalra S, Jain V. Dental complications and management of patients on bisphosphonate therapy: a review article. J Oral Biol Craniofac Res. 2013;3(1):25-30.

20. Elsubeihi ES, Zarb GA. Implant prosthodontics in medically challenged patients: the University of Toronto experience. J Can Dent Assoc. 2002;68(2):103-8.

21. Chrcanovic BR, Albrektsson T, Wennerberg A. Dental implants inserted in male versus female patients: a systematic review and meta-analysis. J Oral Rehabil. 2015;42(9):709-22.

22. Alsaadi G, Quirynen M, Komárek A, van Steenberghe D. Impact of local and systemic factors on the incidence of oral implant failures, up to abutment connection. J Clin Periodontol. 2007;34(7):610-7.

23. Alsaadi G, Quirynen M, Michiles K, Teughels W, Komárek A, van Steenberghe D. Impact of local and systemic factors on the incidence of failures up to abutment connection with modified surface oral implants. J Clin Periodontol. 2008;35(1):51-7.

24. Chrcanovic BR, Albrektsson T, Wennerberg A. Smoking and dental implants: a systematic review and meta-analysis. J Dent. 2015;43(5):487-98.

25. Russell RG, Xia Z, Dunford JE, Oppermann U, Kwaasi A, Hulley PA, et al. Bisphosphonates: an update on mechanisms of action and how these relate to clinical efficacy. Ann N Y Acad Sci. 2007;1117:209-57.

26. Ruggiero SL, Dodson TB, Assael LA, Landesberg R, Marx RE, Mehrotra B. Task force on bisphosphonate-related osteonecrosis of the jaws, American Association of Oral and Maxillofacial Surgeons. Aust Endod J. 2009;35(3):119-30.

27. Marx RE, Sawatari Y, Fortin M, Broumand V. Bisphosphonateinduced exposed bone (osteonecrosis/osteopetrosis) of the jaws: risk factors, recognition, prevention, and treatment. J Oral Maxillofac Surg. 2005;63(11):1567-75.

28. Chadha GK, Ahmadieh A, Kumar S, Sedghizadeh PP. Osseointegration of dental implants and osteonecrosis of the jaw in patients treated with bisphosphonate therapy: a systematic review. J Oral Implantol. 2013;39(4):510-20.

29. Madrid C, Sanz M. What impact do systemically administrated bisphosphonates have on oral implant therapy? A systematic review. Clin Oral Implants Res. 2009;20 Suppl 4:87-95.

30. Gupta S, Gupta H, Mandhyan D, Srivastava S. Bisphophonates related osteonecrosis of the jaw. Natl J Maxillofac Surg. 2013;4(2):151-8.

31. López-Cedrún JL, Sanromán JF, García A, Peñarrocha M, Feijoo JF, Limeres J, et al. Oral bisphosphonate-related osteonecrosis of the jaws in dental implant patients: a case series. Br J Oral Maxillofac Surg. 2013;51(8):874-9. 